\title{
On the Verge of Coalescence: a Dusty Group of Galaxies
}

\author{
S. Temporin and R. Weinberger \\ Institut für Astrophysik, Leopold-Franzens-Universität Innsbruck, \\ A-6020 Innsbruck, Austria \\ F. Kerber \\ Space Telescope - European Coordinating Facility, ESO, D-85748 \\ Garching, Germany
}

\begin{abstract}
We have found a new, very compact group of galaxies characterized by a median projected separation between galaxies of $5.2 / h \mathrm{kpc}$ and a very low velocity dispersion. All the observed member galaxies show emission line spectra and a disturbed morphology. From these properties it emerges that this compact group is in an extremely advanced stage of evolution.
\end{abstract}

\section{Introduction}

Compact groups of galaxies (CGs) are thought to evolve into isolated bright ellipticals, as suggested by numerical simulations (e.g. Barnes 1989; Athanassoula, Makino \& Bosma 1997), but until now there are very few known examples of elliptical galaxies whose properties are suggestive of a remnant of a merging process of the most luminous members of CGs (Mulchaey \& Zabludoff 1999; Ponman et al. 1994). This is why it is very important to have the opportunity to study a CG in an advanced stage of evolution, on the way to coalescence into a field elliptical galaxy.

We may have found such a link between the CG-stage and the final product of its evolution: CG1720-678 was identified as an unusual object during a survey of the Palomar Observatory Sky Survey plates (Weinberger 1995) and we have now recognized in it several properties indicating an advanced stage of evolution.

The properties designating CG1720-678 as a compact group in the classical sense and at the same time characterizing it as an extreme object are the following:

1. Extreme density: the median projected separation of the group members is $5.2 h^{-1} \mathrm{kpc}\left(h=H_{0} / 100 \mathrm{~km} \mathrm{~s}^{-1} \mathrm{Mpc}^{-1}\right)$. As a comparison we note that the most compact Hickson Compact Group (HCG) has a median projected separation of $6.8 h^{-1} \mathrm{kpc}$ (Hickson 1993).

2. Very low velocity dispersion: $\sigma_{2 D}=67 \mathrm{~km} \mathrm{~s}^{-1}$ and $\sigma_{3 D}=99 \mathrm{~km} \mathrm{~s}^{-1}$, while the median values for HCGs are: $\sigma_{2 D}=200 \mathrm{~km} \mathrm{~s}^{-1}$ and $\sigma_{3 D}=331 \mathrm{~km} \mathrm{~s}^{-1}$ (Hickson et al. 1992). 

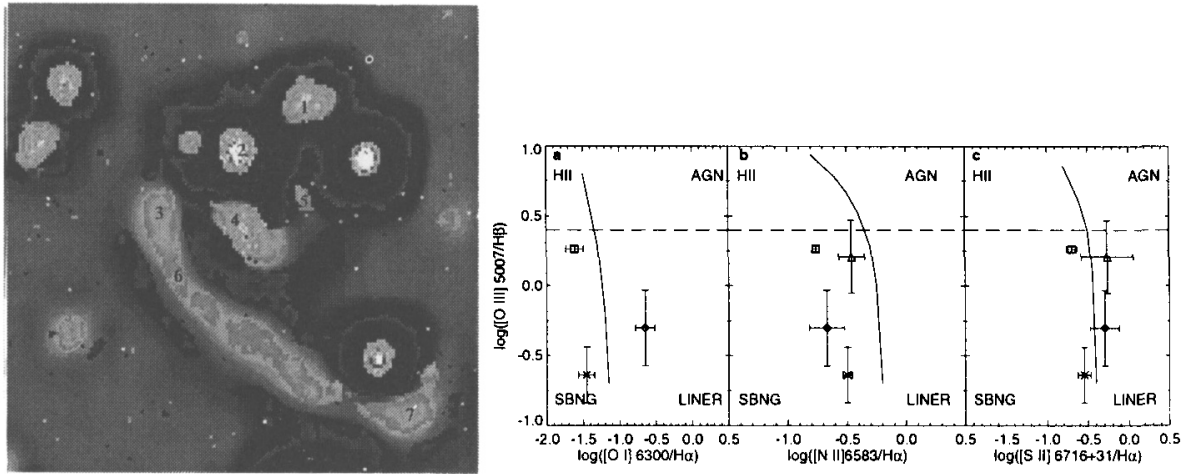

Figure 1. Left: $V$-band image of CG1720-67.8 after Laplacian filtering. Right: Diagnostic diagrams of the four brightest objects of the group; squares, diamonds, triangles and crosses represent the emissionline ratios of objects no. $1,2,3$, and 4 respectively.

3. The four member galaxies of the group are within 3 magnitudes from its brightest galaxy, with $m_{V}$ ranging from $\sim 17.5$ to $\sim 20.5$, as estimated by comparison of our $V$ frame with the images on the SERC $\mathrm{J}$ and ESO R film copies.

4. Isolation: as the nearest catalogued galaxy is about 1 degree away and an inspection of the POSS plates does not reveal the presence of galaxies of comparable magnitude within 11 times the diameter of the group $\left(\sim 30^{\prime \prime}\right)$, the group fulfills the isolation criteria.

\section{Observations and Data Analysis}

Optical imaging and spectroscopy were used to establish the main characteristics of CG1720-67.8: a 900 sec exposure in the $V$ band with a resolution of 0.259 arcsec/pixel and a long-slit spectrum with spatial resolution 0.56 arcsec/pixel, dispersion $2 \AA /$ pixel over the range $3770-7180 \AA$, and spectral resolution $\sim 4$ $\AA$ were obtained at the Du Pont $2.5 \mathrm{~m}$ telescope of Las Campanas Observatory (February 1998); another long-slit spectrum with spatial resolution 0.26 arcsec/pixel, dispersion $2 \AA /$ pixel over the range $3850-8000 \AA$ and spectral resolution $\sim 12 \AA$ was obtained at the MPI $2.2 \mathrm{~m}$ telescope in La Silla (May 1997). After the usual reduction steps, a Laplacian filtering procedure (Richter et al. 1991) was applied to the $V$-frame in order to suppress the noise and enhance faint details. The result is shown in Fig. 1. The spectra, reduced in a standard way and flux-calibrated by means of spectrophotometric standard stars, covered the objects 1 to 6 in Fig. 1.

The three brightest members of the group (no. 2, 4, and 1) can apparently be classified as elliptical galaxies, but an analysis of the filtered image reveals that two of them have disturbed morphologies: no. 4 reveals a possible double nucleus, outer structures that could indicate that it is the product of a merging 
of two disk galaxies, and a faint tail ending in an emission knot (no. 5) whose nature is still unclear. Object no. 1 shows some internal structure, perhaps a double nucleus. An outstanding "arc" dominates the image of the group and is characterized by brightness enhancements at its ends (no. 3 and 7), where the northern one is the fourth-brightest member-object. The arc is probably a tidal feature generated by interaction and/or merging processes.

Spectra of the objects no. 1 to 5 and a portion of the arc (no. 6) were extracted: all of them show emission lines and are characterized by blue continua typical of young stellar populations, except for object no. 2 whose spectrum suggests a mixed stellar population and could be indicative of a post-starburst phase.

Internal extinction estimated from the Balmer decrement after having taken into account the effect of stellar absorption lines (Table 1) seems to indicate a large amount of obscuration (the estimated Galactic foreground extinction is only $\left.\mathrm{A}_{V} \sim 0.3\right)$.

A classification of the type of activity of the four observed galaxies (Fig. 1, right), obtained by means of diagnostic diagrams (Veilleux \& Osterbrock 1987; Coziol et al. 1998), shows that all of these galaxies have a starburst nucleus. Star formation rates per unit projected area have been derived from $\mathrm{H} \alpha$ luminosities (Table 1). The values are comparable or even higher than typical SFR of interacting spiral galaxies (Bushouse, 1987), ranging from $6.11 \times 10^{-11}$ to $7.6 \times 10^{-8} M_{\odot} \mathrm{yr}^{-1} \mathrm{pc}^{-2}$.

Table 1. Estimated non-galactic foreground extinction values $\left(A_{V}\right)$, and star formation rates (SFR) in units of $10^{-8} \mathrm{M}_{\odot} \mathrm{yr}^{-1} \mathrm{pc}^{-2}$.

\begin{tabular}{ccc}
\hline Object & $\mathrm{A}_{V}$ & SFR \\
\hline 1 & $0.8 \pm 0.1$ & 15.0 \\
2 & $1.6 \pm 1.2$ & 7.3 \\
3 & $3.2 \pm 1.4$ & 1.2 \\
4 & $2.0 \pm 0.4$ & 9.9 \\
\hline
\end{tabular}

Puzzling enough, in spite of the high degree of interaction, the enhanced star formation activity, and the considerable amount of extinction, there is no catalogued IRAS source at the position of CG1720-67.8. After building contour maps of the emission in the four IRAS bands, we found only a weak emission, at the $4 \sigma$ level above the background, at $60 \mu \mathrm{m}$. An improved resolution map, obtained after application of the Maximum Entropy method, shows that this emission is peaked at the position of the compact group (Fig. 2).

\section{Discussion}

CG1720-67.8 is extremely compact, presents a high degree of interaction among its members and is dominated by a tidal-like feature. Velocity data derived from the spectra of the brightest members reveal a very low velocity dispersion. All the observed members have emission-line spectra indicating enhanced star formation activity, which we interpret to be a consequence of ongoing interactions. 

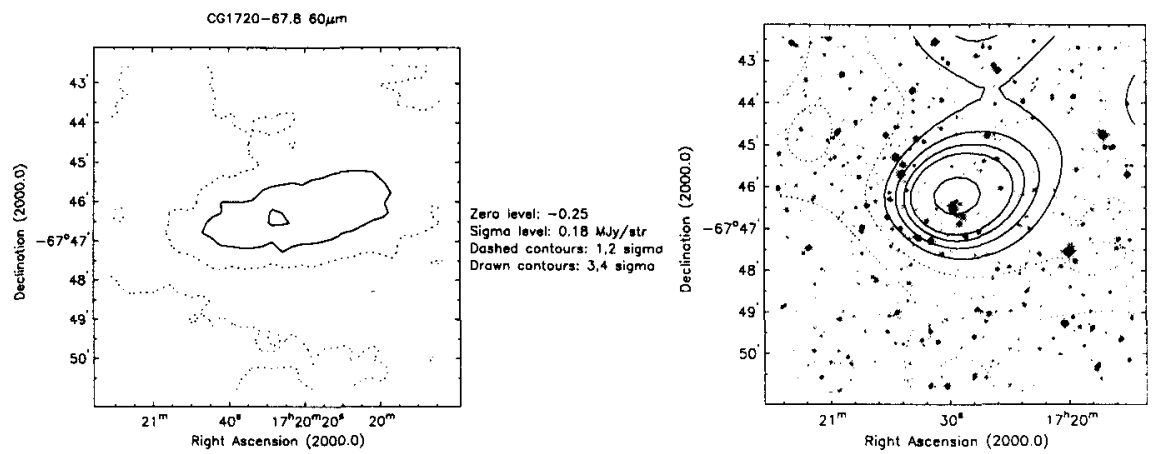

Figure 2. $\quad 60 \mu \mathrm{m}$ IRAS map (left); Maximum Entropy result overlaid to the DSS image of the group (right).

Traces of a post-starburst phase may be present in the brightest object. All of these properties lead to suppose that the group is on the verge of coalescence.

The most puzzling question is represented by the lack of detected FIR emission in spite of the enhanced star formation activity and of the great amount of (non-Galactic) foreground obscuration. We suggest as a possible explanation that the strong extinction is due to cool dust, probably emitting beyond the IRAS bands, that could have been blown off far away as a consequence of strong galactic winds.

We believe that this group represents an important stage of the CGs' evolution and is worthy of further detailed studies.

Acknowledgments. We are grateful to G. Richter for making available to us the Potsdam Image Processing System, and to S. Ciroi for providing us with the Maximum Entropy IRAS map. The IAU kindly assisted with a travel grant.

\section{References}

Athanassoula, E., Makino, J., \& Bosma, A. 1997, MNRAS, 286, 825

Barnes, J. E. 1989, Nature, 338, 123

Bushouse, H. A. 1987, ApJ, 320, 49

Coziol, R., Ribeiro, A. L. B., de Carvalho, R. R., \& Capelato, H. V. 1998, ApJ, 493,563

Hickson, P. 1993, Astrophys. Lett.\& Comm., 29, 1

Hickson, P., Mendes de Oliveira, C., Huchra, J. P., \& Palumbo, G. C. 1992, ApJ, 399, 353

Mulchaey, J. S., \& Zabludoff, A. I. 1999, ApJ, 514, 133

Ponman, T. J., Allan, D. J., Jones, L. R., Merrifield, M., McHardy, I. M., Lehto, H. J., \& Luppino, G. A. 1994, Nature, 369, 462

Richter, G. M., Lorenz, H., Böhm, P., \& Priebe, A. 1991, Astron. Nachr., 312, 345 
Veilleux, S., \& Osterbrock, D. E. 1987, ApJS, 63, 295

Weinberger R. 1995, PASP, 107, 58 